\title{
Zwischen «Wahrung des Besitzstands» und Forderungen: Antwort auf einen Leserbrief
}

Lieber Herr Kollege Schlaginhaufen

Wir veröffentlichen praktisch alle Leserbriefe, die bei der Redaktion eingehen, selbstverständlich ohne sie in irgendeiner Form zu zensieren. Zu einigen publizieren wir jeweils mit dem Einverständnis des Verfassers eine Antwort, eine kurze Erläuterung oder eine Stellungnahme des Zentralvorstands.

Ihr Brief (S. 1752) ruft durch seinen Tonfall und die darin vertretenen Ideen zum Dialog auf. Daher möchte ich hier direkt auf ihn eingehen, womit Sie sich freundlicherweise einverstanden erklärt haben.

Hier also meine Antwort: Sie befürchten, dass Flexibilität in bezug auf unsere Standpunkte, unsere Haltung und die Art und Weise, wie wir unsere Grundsätze umsetzen, dazu führt, dass wir uns als «gute Ärztinnen und Ärzte», die sich angesichts der Bedürfnisse und Erwartungen der anderen zurücknehmen, dem Zeitgeist unterwerfen.

Doch genau das Gegenteil ist der Fall. Flexibel sein und sich anpassen können und wollen ist für die Ärzteschaft ein grundlegender Aspekt, der uns ermöglicht, in der Gesellschaft unseren eigentlichen Platz als Ärzte und Fachleute für die Betreuung von Menschen zu bewahren und geltend zu machen.

Selbstverständlich ist das Eintreten für unsere Arbeitsbedingungen, einschliesslich unserer finanziellen Interessen, von grundlegender Bedeutung. Dies ist eine der zentralen Aufgaben der FMH, für die wir uns vorbehaltlos einsetzen. Doch um diese Interessen gut zu vertreten, müssen wir eine verständliche Sprache sprechen und vor allem über stichhaltige Argumente verfügen, die auf die Anliegen unserer derzeitigen Gesprächspartner Bezug nehmen.

Daher möchte ich nochmals betonen, dass die «Wahrung des Besitzstands» kein Ziel an sich sein kann. Wir müssen unser gesellschaftliches und politisches Umfeld berücksichtigen, damit wir unsere Forderungen verständlich und überzeugend rechtfertigen können. Wir müssen also den Boden vorbereiten und ebnen oder gar zunächst das Argument der «Besitzstandswahrung» aufgeben, um wieder zu den eigentlichen Begründungen vorzustossen, die unsere Forderungen rechtfertigen. Erst nach einer derartigen Abstützung kann der «Besitzstand» wieder in die Diskussion eingebracht werden!

Mit anderen Worten: Wir müssen und können uns weiterentwickeln, ohne befürchten zu müssen, unsere Seele zu verlieren. Doch dazu dürfen wir uns nicht in erster Linie an das klammern, was wir haben, und somit in Unbeweglichkeit verharren. Vielmehr müssen wir uns auf das ausrichten, was wir wollen. Und vor allem müssen wir wissen, weshalb wir es wollen, und dies auch kommunizieren können.

Denn es liegt, wie Sie sehr richtig sagen, ein wesentlicher Teil des Wissens bei uns. Wir kennen uns in der Medizin aus, wir wissen, unter welchen Voraussetzungen ihr hohes Qualitätsniveau gewahrt werden kann, und wir stehen vor Ort mit den Patienten in Kontakt.

Unsere Erfahrung rechtfertigt somit bei weitem den Platz, den wir in den Diskussionen in unserer Gesellschaft beanspruchen, und liefert uns auch die erforderlichen Argumente, um erfolgreich für unsere Arbeitsbedingungen und unsere grundlegenden Prinzipien einzutreten.

Doch damit wir uns auf diese Erfahrung und diese Grundsätze berufen können, müssen wir fähig sein, uns anzupassen, müssen eine verständliche Sprache sprechen und dürfen uns nicht damit begnügen, uns auf Erreichtes abzustützen.

Das ist schwierig, aber notwendig und spannend!

Dr. med. Jacques de Haller, Präsident der FMH 